\title{
Aiding Planning in Air Traffic Control: An Experimental Investigation of the Effects of Perceptual Information Integration
}

Peter M. Moertl, University of Oklahoma, Norman, Oklahoma, John M. Canning, White Oak Technologies, Inc., Silver Spring, Maryland, Scott D. Gronlund, University of Oklahoma, Norman, Oklahoma, Michael R. P. Dougherty, University of Maryland, College Park, Maryland, Joakim Johansson, Ericsson AB, Stockholm, Sweden, and Scott H. Mills, SBC Technology Resources, Inc., Austin, Texas

Prior research examined how controllers plan in their traditional environment and identified various information uncertainties as detriments to planning. A planning aid was designed to reduce this uncertainty by perceptually representing important constraints. This included integrating spatial information on the radar screen with discrete information (planned sequences of air traffic). Previous research reported improved planning performance and decreased workload in the planning aid condition. The purpose of this paper was to determine the source of these performance improvements. Analysis of computer interactions using loglinear modeling showed that the planning interface led to less repetitive - but more integrated - information retrieval compared with the traditional planning environment. Ecological interface design principles helped explain how the integrated information retrieval gave rise to the performance improvements. Actual or potential applications of this research include the design and evaluation of interface automation that keeps users in active control by modification of perceptual task characteristics.

\section{INTRODUCTION}

Air traffic control equipment has changed in recent years as the Federal Aviation Administration (FAA) has adapted its procedures to the growing volume of air traffic across the country. However, two major components of control equipment have remained constant: Specifically, generations of air traffic controllers have utilized a radar screen and flight progress strips as separate representations of aircraft entering their controlled sector and have cognitively integrated those representations. This equipment has proven to be highly beneficial and therefore forms the foundation from which any innovation to the air traffic control system should begin.

If controllers are to manage the increasing volumes of air traffic, planning will be of in- creasing importance. This is evidenced by recent efforts to provide controllers with planaiding technology - for instance, the User Request Evaluation Tool (URET, Arthur \& McLaughlin, 1998), Center-TRACON (Terminal Radar Approach Control) Automation System (CTAS, Denery \& Erzberger, 1995), and En Route Air Traffic Organizer (ERATO, Bressolle, Benhacene, Boudes, \& Parise, 2000). Such interfaces offer additional functions to the controller, such as conflict detection algorithms (URET); automated traffic advisory functions for descending, sequencing, and spacing aircraft (CTAS); and decision aid tools such as filtering options and problem reminders (ERATO). The approach we took in this study was to enhance plan-aiding technology by identifying essential informational elements

Address correspondence to Scott D. Gronlund, University of Oklahoma, Department of Psychology, 455 West Lindsey St., Norman, OK 73019; sgronlund@ou.edu. HUMAN FACTORS, Vol. 44, No. 3, Fall 2002, pp. 404-412. Copyright (C) 2002, Human Factors and Ergonomics Society. All rights reserved. 
that support air traffic planning and determining the extent to which air traffic planning could be improved by optimizing the representation of that information.

Air traffic controllers manage a complex flow of aircraft through their airspace. They maintain strict rules of separation between aircraft while allowing all aircraft to reach their destinations as safely and expeditiously as possible. In planning the routes for the aircraft, two forms of planning can be distinguished. Controllers make tactical plans when they make decisions that relate to the current moment; these plans involve the separation of (usually) pairs of aircraft that could soon violate the separation rules and, hence, need immediate action. They make strategic plans when their plans span longer periods (about 10 min or longer) and typically involve multiple aircraft. An examination of strategic planning in air traffic control is timely, given the concepts being proposed for the future. For instance, the creation of a strategic controller position has been discussed (N. Lawson \& K. Thompson, personal communication, December 15, 1997; see also Vivona, Ballin, Green, Bach, \& McNally, 1996). The proposal provides for one person who would be responsible for a multiple-sector airspace, make decisions about traffic in that airspace, and delegate responsibility for tactical decisions to sector-level controllers. A goal of our project was to develop interface tools for a strategic controller position.

Dougherty, Gronlund, Durso, Canning, and Mills (1999) studied how air traffic controllers make strategic plans for en route traffic (highaltitude, high-speed traffic between destinations) using the radar screen and paper flight progress strips. They identified aircraft sequencing for approach to a common destination as a strategic planning task by analyzing controller verbalizations and using flight progress strips. The specific sequence for a group of aircraft is determined by many factors, such as aircraft speed, altitude, destination, and airspace restrictions. Therefore, sequencing aircraft was a complex cognitive task that involved the consideration of many aircraft over an extended period. Dougherty et al. argued that controllers could benefit from an interface that supported planning the sequences of aircraft.
We begin by outlining the relevant aspects of the traditional air traffic control environment that guided our interface design. Following that, we describe the electronic planning aid and outline its design principles. Finally, we report the evaluation of the planning aid by comparing participants' planning performance using the interface with their performance using the traditional air traffic control environment.

\section{Traditional En Route Air Traffic Control Environment}

Air traffic controllers primarily use information from two different sources: the radar screen and the flight progress strips. The radar screen shows the spatial position and progress of aircraft together with some characteristics of the controlled sector (e.g., boundaries and airways). The radar screen displays the spatial location of aircraft as well as the most vital flight information (identifiers, altitude, speed, and sometimes flight destination). Discrete information about an aircraft (e.g., its destination, flight origin, planned altitude) can be read from the strips (see FAA, 1995) and also is available from the computer readout display. Flight progress strips are small paper strips that are printed from the computer and posted next to the controller about $20 \mathrm{~min}$ prior to arrival of that flight in the sector. Strips also are used to indicate the progress of traffic, as controllers manually update the strips when information about an aircraft changes. In addition, strips carry identifiers that link them to their representations on the radar screen. However, the linkage is not direct: Controllers have to search for the aircraft on the radar screen to coordinate the representations. The radar is of primary importance because it tells the controllers where the aircraft are currently. Strips can be useful for determining the future location of aircraft.

Dougherty et al. (1999) described how controllers use strips for strategic planning purposes. Controllers often classified aircraft into logical groups. These groupings were based on flight plan characteristics (e.g., destination, route, or altitude) and/or flow restrictions. Then strips were ordered to show the planned sequence for these aircraft. In other words, controllers used strips as planning aids with 
which they externally represented their planned sequences. For example, if a controller wanted to plan a sequence of Aircraft A, B, and C, the controller would arrange the three strips in that order. In this way, the strips physically hold the planned sequence of aircraft. In contrast, the radar screen represents the actual air traffic situation. It is important to note that the planned and current sequences of aircraft are not linked to each other and that changes on the radar screen are not reflected by changes to the strips.

The discrepancy between the planned sequence represented by the strips and the positions of the aircraft on the radar screen is essential information for a controller. It makes apparent that something must be done to update the intended path of the aircraft. In order to assess the discrepancy between the planned and current sequence, controllers have to locate the strip and the corresponding aircraft representation on the radar screen and mentally link those representations to each other. In this way, they determine the mismatch between the planned sequence and the current air traffic situation. In other words, the controller is responsible for linking spatial information (location of the aircraft in the sector, distance to other aircraft, distance to specific points in the sector) to discrete information (flight identifier, optimal sequence of aircraft, flight plan, and aircraft type).

In order to help link the spatial and discrete aircraft representations, controllers have to "declutter" the radar screen. A block of information (the data block) is connected to each aircraft on the radar; it contains important flight information about that aircraft. As increasing numbers of aircraft come into a sector, data blocks overlap and become illegible. Therefore, controllers often adjust data blocks into one of eight different positions around the aircraft target and also adjust the distance between the data block and its aircraft. However, some information on the data block is redundant; controllers can get the same information from strips by matching a unique identifier on the strip to the radar. Therefore, decluttering the radar screen to make aircraft distinguishable could be viewed as a hindrance to more important tasks, such as strategic planning. Although controllers can utilize radar decluttering to create memory cues, a perceptually improved interface might supplant the memory demands. The necessity for decluttering seems to be caused by the separation of the different information sources.

We addressed this problem by designing a new interface that took over the task of linking spatial and discrete information. The interface should allow controllers to perceptually assess the difference between the current air traffic sequence and the planned sequence without having to frequently declutter the radar screen. We discuss this electronic planning aid next.

\section{Electronic Planning Aid}

The electronic planning aid changed the task of planning air traffic sequences by perceptually representing a major constraint in the controllers' environment that was not visible in the traditional environment (see Moertl, n.d., for screen shots of the interface). It linked discrete information to its spatial representation on the radar screen. This dynamic linkage between the planning aid and the radar screen was implemented by presenting the participants with two computer screens: Figure 1 shows a snapshot of the two screens.

The planning aid was linked to the radar screen, and the participant could move the cursor between the two screens. Aircraft in a sector were represented on the planning aid by rectangular tokens, as shown in Figure 1. The tokens contained the aircraft's identification number, destination airport, equipment type, and altitude. Aircraft tokens were marked and unmarked by clicking on them, in which case a check mark symbol appeared or disappeared on the token. All aircraft were represented on both screens and could be perceptually located on one screen by moving the cursor over the token on the planning aid or by clicking on its target on the radar screen.

The planning aid allowed participants to categorize aircraft and updated that representation on the radar screen in the color corresponding to their category. Figure 1 shows two aircraft (UAL 755 and DAL80) that a participant placed into different categories. Because each column of aircraft was in different colors, participants did not have to encode and 


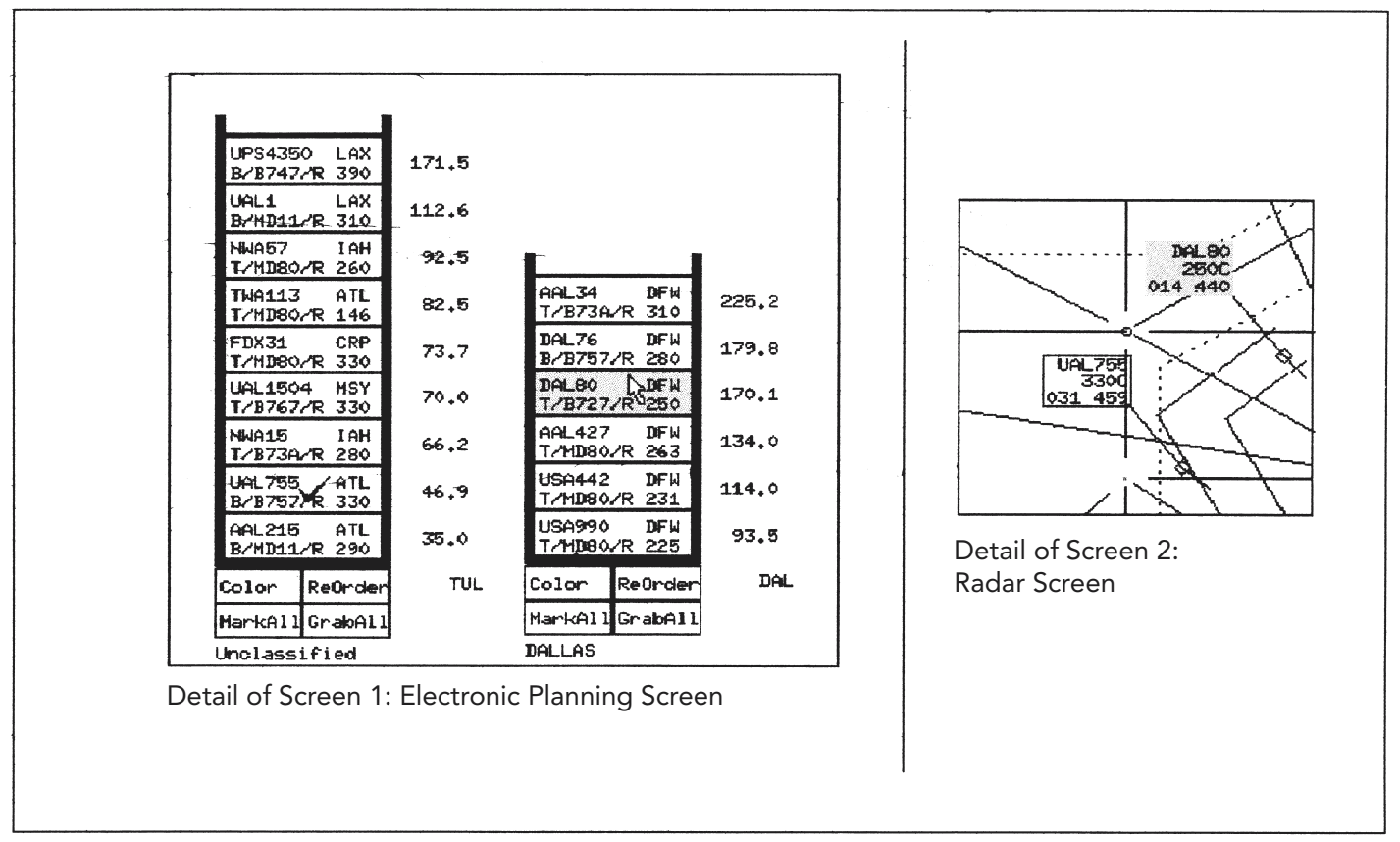

Figure 1. The dynamic linkage between the planner screen and the radar screen. On the radar, every diamond-shaped aircraft representation is linked to a data block of flight information (in order from top, left to right: aircraft identification, altitude in 100 feet, computer identification number, and speed). The two aircraft on the radar screen (UAL755 and DAL80) are grouped into different categories of traffic (i.e., final destination "Dallas" and "unclassified"). Different categories of traffic are represented in different colors. Aircraft are selected by clicking on them (UAL755). Moving the cursor over any token on the planning screen or a target on the radar screen puts a rectangle around the two representations (DAL80).

remember the categories of each aircraft after they were categorized because they were represented perceptually on the radar screen.

The planning aid also allowed the participant to automatically sort categorized air traffic according to time or distance to specific points (fixes) along the route. This information was displayed adjacent to each aircraft token. Note that this automatic sorting did not include higher-level conflict information; it simply was based on distance/time measures and therefore provided only an initial approximation of aircraft order. These initial sequences needed manual updating and checking. Participants also could get information about distance between points on the radar screen by using a distance-measuring tool. Use of this tool was similar to how controllers measure distance on traditional radar screens.

One essential consequence of this design was that a planned sequence could be perceptually compared with the current sequence by sliding the cursor across the sequence on the planning screen. This allowed the controller to observe how the sequenced position of each aircraft corresponded to its current position on the radar screen. Any discrepancy between the planned sequence and the current sequence was therefore made perceptually salient to the controller. If the planned sequence differed from the current sequence, this discrepancy signaled the need for modifications either to the planned sequence or to an aircraft's path. The discrepancy represented an important constraint, as it guided the controller toward those aspects of the situation needing control interventions.

Do participants rely on the radar screen to the same extent when using the planning aid as when using the strips? The answer to this question should provide evidence of the extent to which participants made differential usage of the same radar functions in each condition. For example, they should not have to declutter 
the radar as much. From observed differences, we can infer how participants used and accessed information differently in the two conditions.

\section{METHOD}

Twelve en route air traffic controllers participated in the experiment. All participants were full-performance-level controllers who served as instructors at the FAA Academy. Full-performance-level controllers are qualified to control traffic in a sector by themselves. The experiment was conducted at the FAA Civil Aeromedical Institute in Oklahoma City. All participants were familiar with the airspace (i.e., sector) used in the experiment.

Each of the 12 participants was given the task of planning and maintaining aircraft sequences for a generic en route sector and to communicate the sequence orally to a tactical controller (tactician) who implemented it. The tactician, another en route air traffic controller, was the subject-matter expert. All participants performed the same task under two conditions: (a) using the electronic planning aid without strips (planner condition) and (b) using paper flight strips without the electronic planning aid (strips condition). Participants used the same radar screen in both conditions, although some of the radar functionality was absent without the planning aid (i.e., the color coding of aircraft). The two scenarios were busy; up to 44 aircraft were in the sector during $20 \mathrm{~min}$ and included arrivals, departures, and overflights. The order of the scenarios and the order of the two conditions (planner or strips) were counterbalanced across participants.

Each participant completed a 45-min training session on the use of the planning aid and radar screen. One or 2 days later, each participant completed a 1 -h practice session. No training was given on the use of the flight progress strips, as all participants had extensive experience with them in the field. The experiment started 1 or 2 days following the practice session. Immediately prior to testing, participants practiced with the planning aid for a few minutes to refamiliarize themselves with its operation.

For each scenario, the participant was told about any special airspace restrictions in effect and was given sufficient time to set up either the planning aid or paper strips while the aircraft in the scenario remained paused. During this time they verbally conveyed their plan to the tactician. The simulation was started after the plan was conveyed. The simulation ran for $20 \mathrm{~min}$, with a brief pause after $10 \mathrm{~min}$.

\section{RESULTS AND DISCUSSION}

We have previously described the details of the participants' superior performance and reduced workload when using the planning aid (see Canning, Johansson, Gronlund, Dougherty, \& Mills, 1999; Moertl, Canning, Johansson, Gronlund, Dougherty, \& Mills, 2000). The advantage for the planning aid condition was especially noteworthy because participants had, on average, about 10 years' experience with paper strips and less than $2 \mathrm{~h}$ of practice with the planning aid. In the present study, we were interested in the differential usage of the radar screen between the conditions and what that could tell us about why the planning aid led to superior planning performance and reduced workload.

\section{Log-Linear Modeling of User Interactions}

Olson, Herbsler, and Rueter (1994) presented log-linear modeling as a technique for analyzing human-computer interactions. Log-linear models allow the test of main effects and interactions of models that fit empirical humancomputer interaction frequencies and thereby allow the determination of sequential structure and structure changes between conditions. Olson et al. used log-linear modeling on a set of interaction data that were semantically categorized by human judges. Such a procedure can prove highly useful but has the disadvantage of costing considerable time resources for the manual categorizations of actions. In contrast, we used this approach to analyze raw data files that were not categorized by human judges. We therefore sidestepped the objection that the analysis of categorized data might reflect the judgments of human judges in addition to actual empirical patterns in the computer interactions. In contrast to Olson et al., our design included repeated measurements for which we accounted in our models. 
TABLE 1: Observed and Expected Frequencies for a Log-Linear Model Assuming No Difference between Experimental Conditions

\begin{tabular}{lcccc}
\hline & $\begin{array}{l}\text { Frequency } \\
\text { in Strip } \\
\text { Condition }\end{array}$ & $\begin{array}{l}\text { Frequency } \\
\text { in Planner } \\
\text { Condition }\end{array}$ & $\begin{array}{l}\text { Predicted } \\
\text { Frequency }\end{array}$ & $\begin{array}{l}\text { Standardized } \\
\text { Residual for } \\
\text { Strip } \\
\text { Condition }\end{array}$ \\
\hline Adjust vector length & 3 & 6 & 4.5 & -0.71 \\
Invalid command (error) & 72 & 83 & 77.5 & -0.62 \\
Zoom in/out & 10 & 11 & 10.5 & -0.15 \\
Move information table & 2 & 2 & 2 & 0.00 \\
Altitude filter & 12 & 10 & 11 & 0.30 \\
Invalid track (error) & 3 & 2 & 2.5 & 0.32 \\
Select token & 482 & 408 & 445 & $1.75^{\mathrm{a}}$ \\
Distance measurement & 108 & 56 & 82 & $2.87^{\mathrm{a}}$ \\
Data block adjustment & 661 & 520 & 590.5 & $2.90^{\mathrm{a}}$ \\
\hline
\end{tabular}

a Observed frequency is outside a $95 \%$ confidence interval around the expected frequency.

Although participants interacted with the radar screen more frequently in the strip condition - significant main effect of experimental condition, Wald $\chi^{2}(1)=17.07, p<.01-$ of primary interest was determining the source of these differences. We compared the observed frequencies with the expected frequencies and determined how participants interacted with the radar screen differently in the two conditions.

Participants performed nine different types of actions on the radar screen (listed in Table 1). We compared observed frequencies for each action with expected frequencies assuming no differences between conditions (i.e., standardized residuals for each action and condition). The standardized residuals were calculated as the difference between the predicted and observed frequency divided by the square root of the predicted frequency. Table 1 displays the results of this analysis; the last column shows the standardized residuals. The model predicted the observed frequencies satisfactorily (within a 95\% confidence interval) for all but three of the actions. This meant that these three actions occurred with differing frequencies in the two conditions. We discuss these three user actions in turn.

Select token. Participants selected significantly more aircraft on the radar screen in the strip condition than in the planning aid condi- tion. Selecting an aircraft creates a border around its data block, which enhances its visibility. Participants in the planning aid condition did not need this perceptual aid as frequently, presumably because they could rely on the dynamic linkage between the two screens to perceptually locate aircraft on the radar screen.

Distance measurement. Participants measured the spatial distance between points on the radar screen more frequently in the strip condition. Distance information was crucial for planning, as it allowed estimation of when aircraft would reach specific points in the sector. Participants in the planning aid condition did not measure the distances on the radar screen as frequently, presumably because they could rely on the time/distance information that was presented to them next to each aircraft on the planning aid.

Data-block adjustment. Data-block adjustments included changing and adjusting datablock position. Participants adjusted data blocks more frequently in the strip condition than in the planning aid condition. As mentioned, controllers declutter their radar screen in order to make data-block information visible. These adjustments are an important index of the usage of the radar screen. In the strip condition, participants had to get their flight information from the radar screen and had to 
declutter the radar screen to get to this information. However, when using the planning aid, participants adjusted the data-block position less frequently and instead relied on the planning aid to review flight information. This was consistent with the greater ease of information access in the planning aid condition and the increased time spent in the strips condition on "housekeeping" functions.

Participants interacted with the radar screen less when they worked with the planning aid. They manipulated aircraft less (visually highlighted or marked aircraft less) and adjusted data blocks less often. They also assessed spatial distance less often. The greater ease of accessing information was the result of replacing radar functionality with the functionality of the planning aid. The prior results examined interaction frequencies. In what follows, sequences of user interactions were examined to provide additional insights into how participants used the radar screen differently between the two conditions.

\section{User Interactions Sequence Analysis}

Differences in sequences of interactions between conditions were also testable using log-linear modeling. For each condition, the empirical probabilities of an interaction occurring after another interaction were compared with the estimated probabilities, assuming there were no differences between conditions. In a log-linear model, the difference between condi- tions was tested by a three-way interaction of the main factors (antecedent action, consequent action, and experimental condition; see Olson et al., 1994). We found a significant three-way interaction, Wald $\chi^{2}(13)=610.97$, $p<.01$, which indicates different, nonrandom sequences between the conditions. Accordingly, we found significant differences by comparing specific action sequences between conditions. Again, we calculated the standardized residuals using antecedent and consequent actions and their interaction. Because there were 115 empirically occurring sequences, only the 4 sequences that were not predicted by the model are reported in Table 2 . These differences reinforced the usage differences between the conditions noted earlier in this paper.

Participants had more frequent repetitions of distance measurement and data-block adjustment in the strip condition. They also cycled between adjusting data blocks and selecting aircraft, and vice versa, more frequently in the strip condition. Information retrieval in the strip condition appeared more repetitive compared with that in the planning aid condition. Participants needed to declutter the radar screen more because they had to integrate the strips with the aircraft on the radar screen. Furthermore, the integration effort seemed not to occur as an isolated event; rather, it happened in bursts of repeated decluttering activity. It is hard to see how these bursts of decluttering

TABLE 2: Sequences of User Actions

\begin{tabular}{lllll}
\hline $\begin{array}{l}\text { Sequence } \\
\text { of User } \\
\text { Actions }\end{array}$ & $\begin{array}{l}\text { Frequency } \\
\text { in Strip } \\
\text { Condition }\end{array}$ & $\begin{array}{l}\text { Frequency } \\
\text { in Planner } \\
\text { Condition }\end{array}$ & $\begin{array}{l}\text { Frequency } \\
\text { Predicted }\end{array}$ & $\begin{array}{l}\text { Standardized } \\
\text { Residual for } \\
\text { Strip } \\
\text { Condition }\end{array}$ \\
\hline $\begin{array}{l}\text { Repeated distance } \\
\text { measurement }\end{array}$ & 60 & 14 & 37 & $3.78^{\mathrm{a}}$ \\
$\begin{array}{l}\text { Repeated data block } \\
\text { adjustment }\end{array}$ & 202 & 129 & 165.5 & $2.83^{\mathrm{a}}$ \\
$\begin{array}{c}\text { Aircraft selection - } \\
\text { data block adjustment }\end{array}$ & 406 & 331 & 368.5 & $1.95^{\mathrm{a}}$ \\
$\begin{array}{c}\text { Data block adjustment - } \\
\text { aircraft selection }\end{array}$ & 430 & 361 & 395.5 & $1.73^{\mathrm{a}}$ \\
\hline
\end{tabular}

Note. The positive standardized residuals indicate that user action sequences occurred in the strip condition more frequently than expected by the model.

a Observed frequency is outside a $95 \%$ confidence interval around the expected frequency. 
activity could entail any memory-enhancing function. This manual matching was much less prevalent in the planning condition, in which participants used fewer interactions in absolute numbers and fewer self-repeating interactions to retrieve the necessary information.

\section{CONCLUSIONS AND IMPLICATIONS}

Complex cognitive tasks such as planning the sequencing of air traffic can be supported by integrating different information sources. The planning aid resulted in less repetitive, but more integrated information retrieval, as compared with the traditional planning environment. Less repetitive interactions were possible because the interface itself provided the physical integration of the information. This provided one reason why Moertl et al. (2000) found that planner workload was reduced. The integration of the discrete flight information with the radar information allowed participants to develop their plan 6.3 min faster in the planning aid condition than in the strip condition.

The planning aid not only made planning easier, it also improved the quality of those plans. Moertl et al. (2000) found no difference in plan quality during the first half of the scenario (although those plans were developed much faster). However, in the second half of the scenario, when participants were changing and updating their plans, planning performance was superior in the planning aid condition. The planning aid especially was beneficial in this situation because adapting plans to the current traffic situations depended strongly on the integration of planned sequence information with the current air traffic situation. The planning aid was designed to do exactly that. Participants could see on the radar the sequence of aircraft that they had proposed on the planning aid. By sliding the cursor over the corresponding aircraft on the planner, they could see how the plan was progressing. This visual display of a planned sequence on the radar gave the controller an important indication of where changes were required. Aircraft that were out of sequence and did not "light up" where they should focused the participant on relevant decision points.

The current interface has many characteristics of an ecological interface (e.g., Effken, Kim, \&
Shaw, 1997; Lintern, 2000; Pawlak \& Vicente, 1996; Rasmussen \& Vicente, 1990; Vicente \& Rasmussen, 1990). Ecological interface design argues for a perceptual formulation of user goals within the interface. The interface then facilitates actions as the user perceives his or her goals mirrored in the affordances of the interface. In this way, the interface guides users' interactions without major intrusions or the need for automation. It replaces effortful cognitive processes with parallel, perceptual processes.

Perceptual information integration proved to be a successful design principle when we looked at the cognitive task of planning air traffic in isolation. Future experiments should be directed at integrating strategic planning with other controlling tasks (e.g., tactical planning). Only then can it be determined whether the planning aid can replace strips or if other controller tasks still require the availability of paper flight progress strips. Also, it is important to keep in mind our focus on strategic planning and the accompanying decision to isolate the strategic planning tasks from the tactical planning tasks by assigning these responsibilities to two different individuals. It is possible that a single controller responsible for both tactical and strategic planning would not find the planning aid useful. However, that was not the goal of our project; the goal was to develop an interface for a possible future strategic controller position. A different interface may have resulted if our goal had been to develop an interface to enhance the strategic planning capabilities of a controller working a sector alone. An important next step will be to compare the planning aid with URET and CTAS to determine what aspects of the air traffic control task can best be accomplished through information reorganization and what can be best handled by automation.

Recent research suggested the advantage of active control over passive monitoring in air traffic management (e.g., Metzger \& Parasuraman, 2001). Our findings can be applied to the design and evaluation of interfaces that keep the controller in the loop. Specifically, reliance on perceptual processes could serve as an alternative to outsourcing plan development to a piece of software. This allows the controller to do what humans are good at (parallel perceptual 
processing) while allowing the computer to do what it is good at (organization and linking information). Relatively simple modifications of the perceptual properties of an interface can decrease task difficulty and increase human performance without infringing on higher-level cognition. We recommend that perceptual optimization of interfaces should be accompanied by an empirical analysis of behavioral differences between the new and old interface. As a result, tasks that are best accomplished by a human operator should be delineated from those more appropriately left to a computer. Our findings lead us to believe that the design and evaluation of interfaces would extensively benefit by this process.

\section{ACKNOWLEDGMENTS}

The support of the FAA under agreement 97-G-037 is gratefully acknowledged. We thank Frank Durso of Texas Tech University and Robert Terry of the University of Oklahoma for their methodological expertise and Carol Manning and Scott Shappell from FAA-CAMI for their comments on this manuscript. We also extend our thanks to Ron Andrei and Paul DeBenedittis of the FAA Academy and Duane Duke and Rob Enos of the FAA Office of Information Services for their expertise.

\section{REFERENCES}

Arthur, W. C., \& McLaughlin, M. P. (1998). User request evaluation tool (URET) interfacility conflict probe performance assessment (Tech. Report MP 98W0000204). McLean, VA: Mitre Corp.

Bressolle, M. C., Benhacene, R., Boudes, N., \& Parise, R. (2000, June). Advanced decision aids for air traffic controllers: Understanding different working methods from a cognitive point of view. Paper presented at the 3rd International Air Traffic Management Seminar, Napoli, Italy.

Canning, J. M., Johansson J., Gronlund, S. D., Dougherty, M. R. P. \& Mills, S. H. (1999). Effects of a novel interface design on strategic planning by en route controllers. In Proceedings of the 10th International Symposium on Aviation Psychology (pp. 528-533). Columbus, OH: Ohio State University.

Denery, D. G., \& Erzberger, H. (1995). The center-TRACON automation system: Simulation and field testing (NASA Tech. Memorandum 110366). Moffett Field, CA: NASA-Ames Research Center.

Dougherty, M. R. P., Gronlund, S. D., Durso, F. T., Canning, J. M., \& Mills, S. H. (1999). Plan generation in air traffic control. In Proceedings of the 10th International Symposium on Aviation Psychology (pp. 522-527). Columbus, OH: Ohio State University.

Effken, J. A., Kim, N. G., \& Shaw, R. E. (1997). Making the constraints visible: Testing the ecological approach to interface design. Ergonomics, 40, 1-27.
Federal Aviation Administration. (1995). Air traffic control handbook (7110.65J). Washington, DC: FAA Air Traffic Operations Program.

Lintern, G. (2000). An affordance-based perspective on humanmachine interface design. Ecological Psychology, 12, 65-69.

Metzger, U., \& Parasuraman, R. (2001). The role of the air traffic controller in future air traffic management: An empirical study of active control versus passive monitoring. Human Factors, 43, 519-528.

Moertl, P. M., Canning, J. M., Johansson, J., Gronlund S. D., Dougherty, M. R. P., \& Mills, S. H. (2000). Workload and performance in FOPA: A strategic planning interface for air traffic control. In Proceedings of the XIVth Triennial Congress of the International Ergonomics Association and 44th Annual Meeting of the Human Factors and Ergonomics Society (Vol. 3, pp. 65-68). Santa Monica, CA: Human Factors and Ergonomics Society.

Moertl, P. M., University of Oklahoma Psychology Department. (n.d.). FOPA - Flight organizer planning aid. Retrieved August 15, 2002, from http://www.ou.edu/cas/psychology/ HTIC/FOPA/

Olson, G. M., Herbsler, J. D., \& Rueter, H. H. (1994). Characterizing the sequential structure of interactive behaviors through statistical and grammatical techniques. Human-Computer Interaction, 9, 427-472.

Pawlak, W. S., \& Vicente, K. (1996). Inducing effective operator control through ecological interface design. International Journal of Human-Computer Studies, 44, 653-688.

Rasmussen, J., \& Vicente, K. J. (1990). Ecological interfaces: A technological imperative in high-tech systems. International Journal of Human-Computer Interaction, 2, 93-110.

Vicente, K. J., \& Rasmussen, J. (1990). The ecology of humanmachine systems: II. Mediating "direct perception" in complex work domains. Ecological Psychology, 2, 207-249.

Vivona, R. A., Ballin, M. G., Green, S. M., Bach, R. E, \& McNally, B. D. (1996). A system concept for facilitating user preferences in en route airspace (NASA Tech. Memorandum 4763). Moffett Field, CA: NASA Ames Research Center.

Peter M. Moertl is a senior human factors specialist at Titan Systems Corporation in Washington, D.C. He received his Ph.D. in psychology in 2002 at the University of Oklahoma, Norman, Oklahoma.

John M. Canning is principal engineer at White Oak Technologies, Inc., Silver Spring, Maryland. He received his Ph.D. in computer science in 1991 at the University of Maryland.

Scott D. Gronlund is a professor of psychology at the University of Oklahoma. He received his Ph.D. in psychology in 1986 at Indiana University.

Michael R. P. Dougherty is an assistant professor of psychology at the University of Maryland, College Park, Maryland. He received his Ph.D. in psychology in 1999 at the University of Oklahoma.

Joakim Johansson is a system and software designer at Ericsson AB, Stockholm, Sweden. He received his M.S. in computer science in 2000 at the University of Oklahoma.

Scott H. Mills is a senior member of the technical staff at SBC Technology Resources, Inc., Austin, Texas. He received his Ph.D. in psychology in 1995 at the University of Oklahoma.

Date received: June 21, 2001

Date accepted: July 3, 2002 